\title{
Study of Mechanical Behaviour in Shape Memory Alloys Through Adiabatic Thermal Emission and Acoustic Emission Technique
}

\author{
A.C. Lucia and C. Santulli \\ European Commission, Joint Research Centre, Institute for the Systems, Informatics and Safety, \\ 21020 Ispra, Italy
}

\begin{abstract}
Tensile tests were carricd out on commercially available NiTi alloys in order to study their mechanical characteristics up to thie onset of the superelasticity effect. Two realtime supporting techniques, adiabatic thermal emission and acoustic emission, were employed. Thermal émission allowed to follow local temperature variations in the material due to loading, while acoustic emission was able to detect the propagation of related stress wave. When the material is subjected to repeated loading and provided that test conditions remain the same, compared results from these two techniques could enhance the knowledge of material bchaviour. A difficulty lies however in the choice of experimental conditions apt to a successful application of both techniques. Thermal emission in fact has to te performed during a quasi-adiabatic test, while acoustic emission has different requirements, needing in particular a sufficient loading time to allow stresses distribution. Results are promising as a preliminary assessment and could prospectively provide a persuasive quality discrimination.
\end{abstract}

\section{INTRODUCTION}

The term Shape Memory Alloys (SMA) refers to the group of metallic materials that have the ability to return, through exposure to some higher temperature, to their prior shape after having been plastically deformed. Besides the shape memory effect, SMAs display peculiar mechanical properties due to the crystallographic transformation between austenitic and martensitic phases. Superelasticity is among these properties [1]: JRC is at present involved in a project for the study of possible exploitation of superelasticity effect in SMAs for an application to seismic protection and improvement of stability in historical buildings. Such material is supposed to be used in conditions of constant stress, allowing an energy absorption throughout the element due to the hysteresis area showed by its stress-strain curve. In evaluating such possibility of employment for SMAs, an accurate determination for both elastic limit and range of existence for stress plateau could be provided through adiabatic thermal emission and acoustic emission techniques, applied in real time during the test.

Adiabatic thermal emission in particular has already. shown some capability in appraising the mechanical behaviour and its evolution during tensile tests (2 to 4), leading to the measure of a thermoelastic limit and providing furthermore other indications from temperature local changes during the tests. These indications were proved to be suitable to be compared with those coming from acoustic emission, a technique which was experienced in supplying reliable indications on damage evolution during tensile tests, mainly on composites (5). Acoustic emission applications on SMAs have till now concerned prediction of austenite-martensite transformation and correlation of energy content of the material, sorted out in stress wave activity, with its intimate structure $(6,7)$. 


\section{METHODS AND MATERIALS}

From a NiTi (49.8\%) alloy with a Ms temperature of about $10^{\circ} \mathrm{C}$, dog-bone coupons were cut, whose dimensions in $\mathrm{mm}$. are reported in fig. 1 . These specimens underwent afterwards to a solution heat treatment at $930^{\circ} \mathrm{C}$ for 1 hour. Tensile tests were performed using a ZWICK universal testing machine with a $100 \mathrm{kN}$ load cell and measuring strain by knife extensometers (gauge length $25 \mathrm{~mm}$ ). Coupons were clamped with hydraulic grips by applying a $15 \mathrm{MPa}$ pressure. To realise a reasonable compromise between the characteristics of both thermal and acoustic emission, a 1 $\mathrm{mm} / \mathrm{min}$ cross-head speed was chosen.

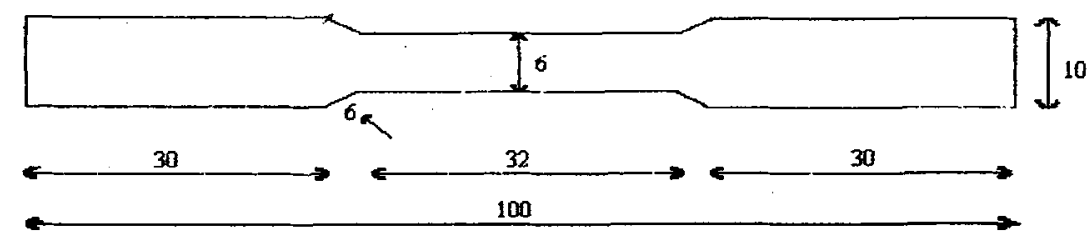

Thickness $=2.4 \mathrm{~mm}$

fig. 1 Dimensions of the specimens

Each specimen was first loaded up to about $70 \%$ of plastic deformation allowed by constant stress plateau, then discharged and loaded again up to its failure. In a first series of 5 specimens adiabatic thermal emission data were acquired, while in a second series both acoustic emission and thermal emission were recorded.

Thermal emission monitoring system was based on DITE acquisition program, described in [4]. Signals from a $30 \Omega$ thermistor placed at the centre of specimen were logged into a $3497 \mathrm{HP}$ unit and amplified, then were treated by DITE, that plots temperature variation against test time, stress or strain. In order to prevent temperature variations from laboratory environment that could affect measurements, a Plexiglas structure surrounded the whole system specimen-grips. DITE did not permit loading to start, if temperature of the specimen was not stable in a range of $\pm 2 \mathrm{mK}$.

Acoustic emission monitoring was instead pcrformed using a commercial system (LOCAN-AT by PAC), treating stress waves detected through a $30 \mathrm{~S}$ microsensor ( $\varnothing$ $5 \mathrm{~mm}$ ), with a $300 \mathrm{kHz}$ peak frequency, placed again at the centre of the specimen. Signals were amplified with a $(30+40) \mathrm{dB}$ gain and filtered on a $100-1200 \mathrm{kHz}$ band.

\section{RESULTS}

In fig. 2 a typical stress-strain curve for the material is shown. From this curve the presence of a constant stress plateau at about $500 \mathrm{MPa}$ is fairly clear; we have to observe however that in no cases was possible to observe the superelasticity phase, because failure occurred just at the end of the plateau or even before, due to surface 
defects induced probably by machining. In effect cracks leading to fracture started in most cases not far from the edges of the specimen grip length.

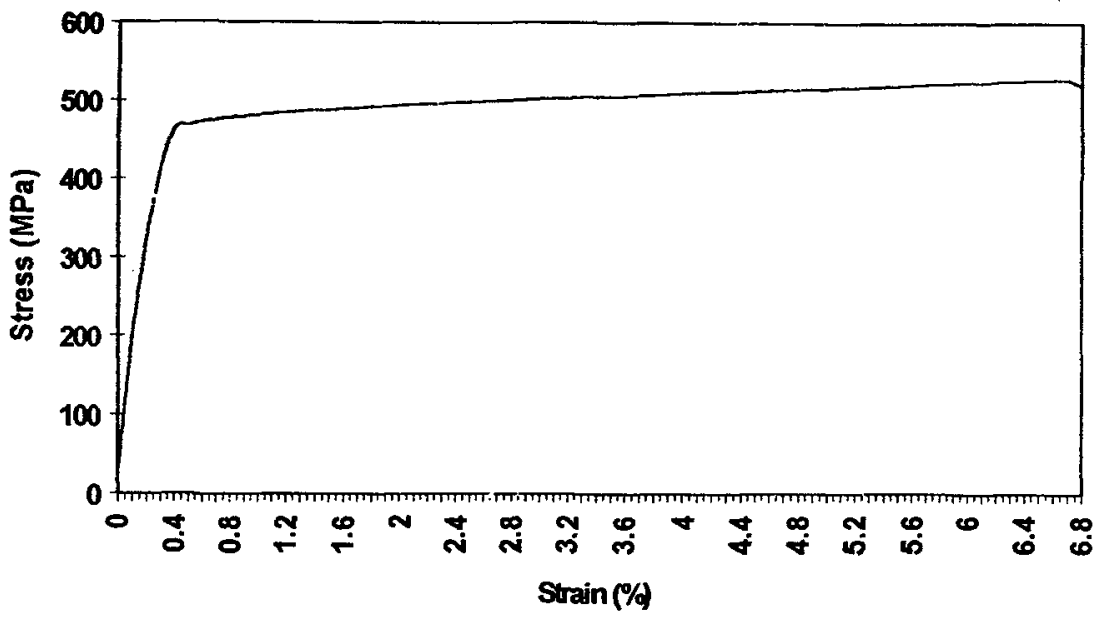

fig.2 Stress (MPa) vs. Strain (\%) curve

Fig. $3 a$ concem the behaviour of the specimens when subjected to the first loading, leading to a plastic deformation of about $5 \%$ and then discharged. During this first loading, we note that local temperature on the specimen centre tends to decrease up to material yielding, then increase because of dislocation movement. This is a well-known phenomenon, that leads to the measure of a thermoelastic limit, that is appreciably coincident with the first elastic limit of the material. Let's consider now the thermal behaviour after yielding. The "knee" of stress-strain curve is accompanied by a considerable temperature increase, whose slope changes with great evidence when the material reaches a constant stress, thus granting a more efficient heat dissipation. The temperature increase is anyway continuous up to the end of loading.

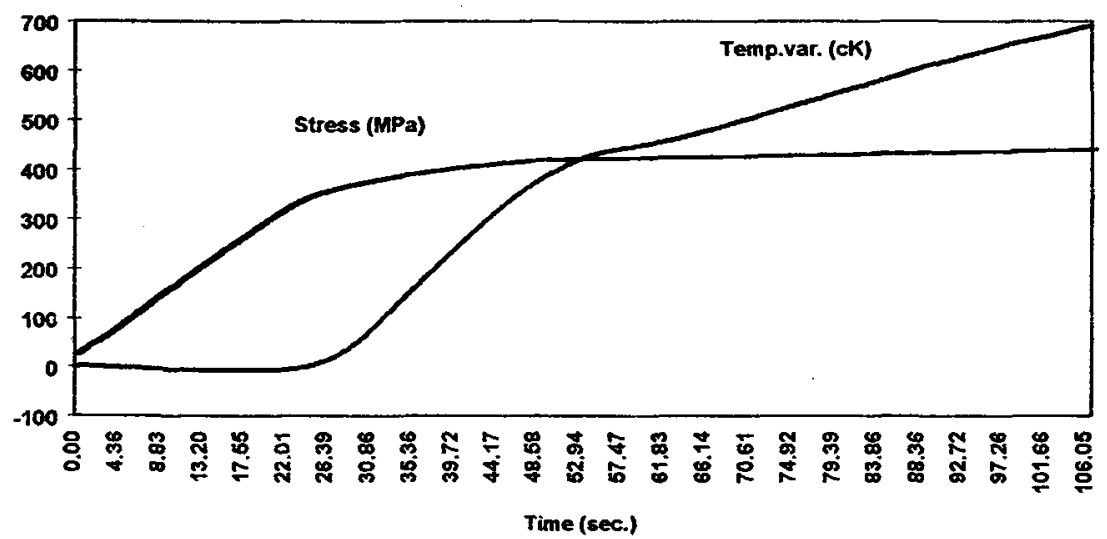

fig.3a Temperature behaviour during first loading of specimen 
Concerning the effect of stress on temperature, A particular attention has nevertheless to be dedicated to the first part of loading, then prior to the thermoelastic limit. In fig. $3 b$ the detail of that phase is reported. Apart from a slight temperature increase just at the beginning of the test, always present and due simply to the first disposition of the specimen to the application of a tensile force, one may note that the temperature decrease is continuous for a certain time (here about $11 \mathrm{sec}$.), followed by a quasiisothermal period. An explanation for that could be that the formation of local plasticization zones have begun to release some energy, able to balance the heat loss due to elastic loading. This can be confirmed from experimental data produced from the second loading up to failure, represented in fig. $4 \mathrm{a}$ and fig. $4 \mathrm{~b}$.

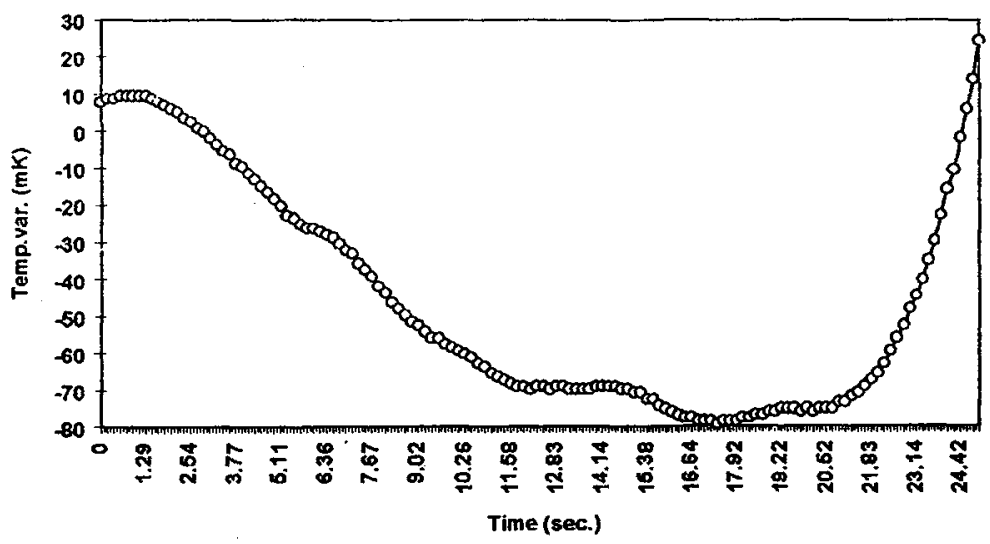

fig. $3 \mathrm{~b}$ Detail of fig. $3 \mathrm{a}$ concerning the first $25 \mathrm{sec}$. of the test

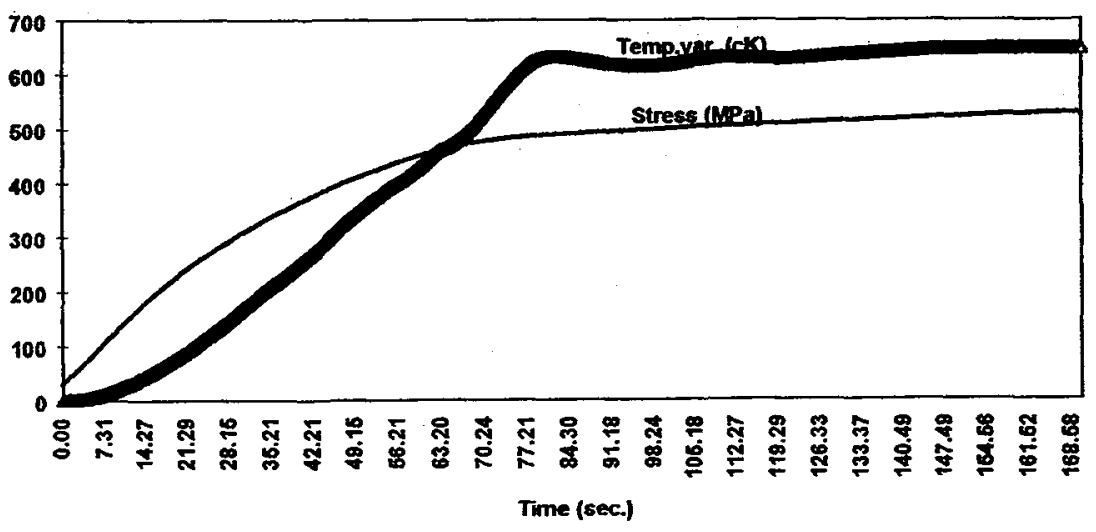

fig.4a Temperature behaviour during second loading of specimen up to failure

The main differences discernible from the first situation are that:

1. No temperature decrease was observed during the elastic loading, as reported with greater details in fig. $4 b$, concerning only the first 15 seconds of the test. This presumably because the importance of local plastic zones is sufficiently important to hide the effect due to hindrances to dislocations motion. 
2. The temperature increase is not continucus and fracture does not produce any perceivable local heating: we assume a relevant heat dissipation from the centre of the specimen, owing to the already mentioned eccentric position of the fatal crack.

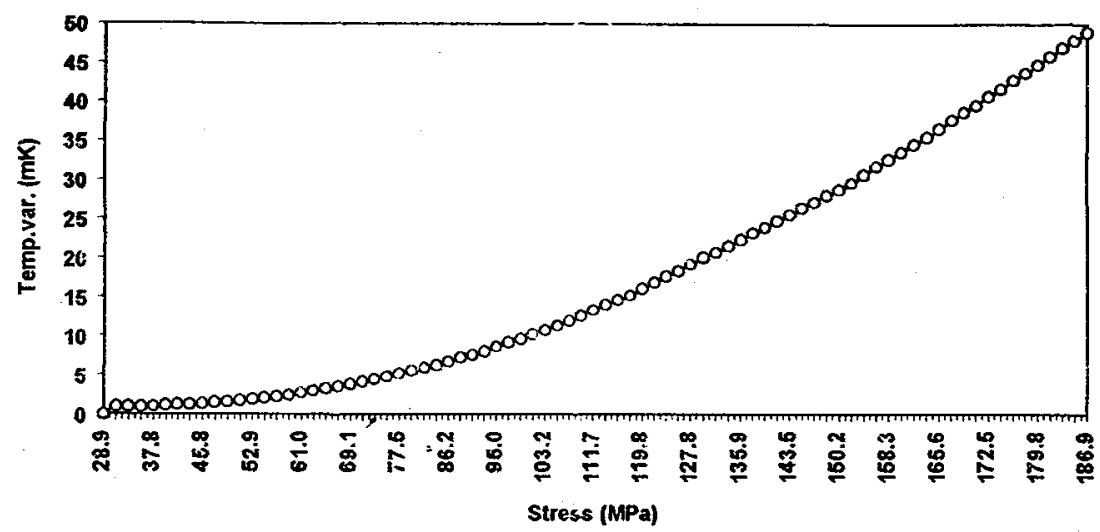

fig. $4 \mathrm{~b}$ Temperature variation vs. stress during the first $15 \mathrm{sec}$. of the second loading

What remains to be lighted is the possible role of acoustic emission in this analysis. Our set-up envisaged the application of a single sensor, both for geometry problems and to reproduce with the greater fidelity the experimental disposition already used during tests monitored with thermal emission. The limitation of this set-up lies of course in the loss of localisation data; attention was therefore paid on cumulative counts curves, whose observation can be generally correlated to onset of plastic deformation and movement of dislocations.

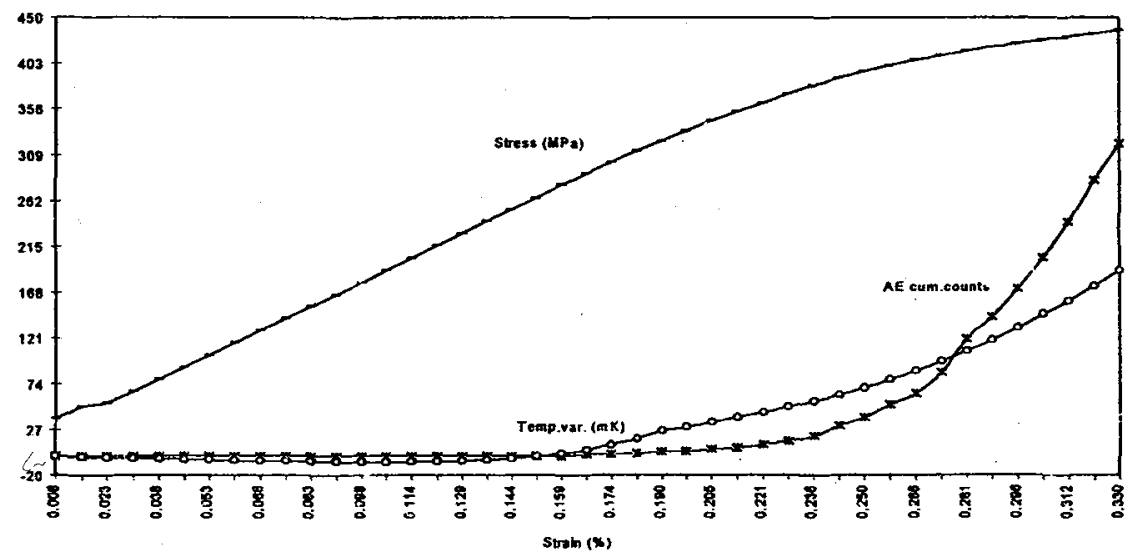

fig. 5 Comparison among stress-strain, acoustic emission and thermal emission curves

In fig. 5 , referred to a specimen loaded for the first time, stress and temperature curves are plotted together with acoustic emission cumulative counts curve vs. strain.

Here acoustic emission cumulative counts curve indicates an exponential increase of activity, as supposed, after yielding point. The first period of the test instead, corresponding to temperature decrease, showed almost no acoustic emission, probably 
because no dislocation movement was acting. Between these two periods, for some time the temperature is stable, as we already discussed, due to the opposite effects of the presence of growing local plasticization zones and to the already blocked dislocation movement. At that time acoustical activity is present, but the curve has a slope much lower than after the yielding.

\section{CONCLUSIONS}

The combined use on shape memory alloys of two real-time monitoring techniques, as adiabatic thermal emission and acoustic emission, during tensile tests, was demonstrated able to complete and characterise information from tensile tests. The presence of plastic zones' and dislocation movements leading to yielding process were recognised with a sufficient reliability, leading also to a more precise elastic limit measurement. A deeper explanation for these evidences should be provided by executing micrographs on fracture surfaces and also by submitting these materials to repeated loads up to the constant stress plateau

\section{Acknowledgements}

We aim to recognise the continuous feedback with Dr. A. Tuissi of CNR-ITM, who supplied materials to be tested in JRC-ISIS laboratories. The technical support of Mr. E. Magistri is also acknowledged.

\section{References}

(1) R. Stalmans, J. Van Humbeeck.

Shape memory. alloys: functional and smart.

Dept. MTM- Katholieke Universiteit Leuven, May 1996

(2) A.D. Melvin, A.C.Lucia, G.P. Solomos.

The thermal response to deformation to fracture of a carbon/epoxy composite laminate Composites science and technology 1993 (vol 46, n.4)

(3) D.E. Oliver.

Stress pattern analysis by thermal emission (SPATE).

Experimental Techniques, March 1988 pp. 3-6.

(4) A.D. Melvin.

The application of thermal emission analysis to damage assessment and material characterisation.

PhD Thesis, Loughborough Univ. of technology, April 1991.

(5) R. Marini, G.P. Solomos.

Composites strength determination via acoustic and thermal emission.

Tech. Note no.I.95.17 ISEI-IE/2860/95, JRC 1995.

(6) E. Vives, J. Ortin, Ll. Mañosa, I. Ráfols, A. Planes.

Distribution of acoustic emission avalanches in martensite transformations.

Journal de physique IV vol.5 February 1995 colloque C2 pp.59-64.

(7) E. Vives, A. Planes.

Spin glass model for the study of avalanches in martensite transformations.

Journal de physique IV vol.5 February 1995 colloque C2 pp.65-70. 